\title{
Desenvolvendo o Pensamento Computacional de Meninas através de Histórias
}

\author{
Juliana B. S. França ${ }^{1,2}$, Beatriz Saburido ${ }^{1}$, Angélica F. S. Dias ${ }^{3,4}$ \\ ${ }^{1}$ Departamento de Computação (DECOMP/UFRRJ) - RJ, Brasil \\ ${ }^{2}$ Programa de Pós-Graduação PPGMMC/UFRRJ e PPGE/UFRRJ - RJ, Brasil \\ ${ }^{3}$ Instituto Tércio Pacitti de Aplicações e Pesquisas Computacionais (NCE/UFRJ), Brasil \\ ${ }^{4}$ História das Ciências e das Técnicas e Epistemologia (HCTE/UFRJ), Brasil \\ \{julibsf, saburido57, angelicafsdias\}@gmail.com

\begin{abstract}
The number of women in the Information Technology field has drastically decreased over the years. However, computing has already been strongly related to women and this paper proposes the rescue of the female audience in computing courses in Brazil, especially in the state of Rio de Janeiro. It presents the initial results of a university extension project where girls between 11 and 16 years old, from public and private schools, are presented to the logical structures of programming through stories and using the Scratch block language. The results achieved show that it is possible to explore technical concepts of programming through stories.
\end{abstract}

Resumo. A presença de de mulheres na área de Tecnologia da Informação tem diminuido drasticamente ao longo dos anos. No entanto, a computação já esteve fortemente relacionada às mulheres e este artigo propõe o resgate do público feminino nos cursos de computação no Brasil, especialmente no estado do Rio de Janeiro. Esta pesquisa apresenta os resultados iniciais de um projeto de extensão universitário onde, meninas entre 11 e 16 anos, de escolas públicas e privadas, são apresentadas às estruturas lógicas de programação através de histórias e usando a linguagem em blocos Scratch. Os resultados alcançados mostram ser possível explorar conceitos técnicos da programação através de storytelling.

\section{Introdução}

O quantitativo de mulheres profissionais na área de Tecnologia da Informação (TI) tem diminuído drasticamente ao longo dos anos, mas nem sempre foi assim. De acordo com [Silva, 2017], existiram mulheres pioneiras que enfrentaram a sociedade para produzir conhecimentos maravilhosos para a informática e tecnologia em geral, como as linguagens de programação, sistemas operacionais, entre outros. Uma delas foi Ada Lovelace que, em 1843, foi a primeira pessoa a desenvolver um algoritmo computacional da história.

No entanto, esse cenário mudou e elas foram desaparecendo das áreas de engenharia e computação. Segundo uma reportagem do Jornal da USP (2018), a primeira turma de Ciências da Computação do IME contava com 20 alunos, sendo 14 
mulheres e 6 homens. Ou seja, 70\% da turma era composta de mulheres. Já a turma de 2016 contava com 41 alunos, sendo apenas 6 meninas, ou seja, 15\%, apontando uma redução de $55 \%$ da participação de mulheres nas turmas do mesmo curso. No mercado de tecnologia brasileira não é diferente, um estudo divulgado pela Softex (2019), aponta que houve queda na participação das mulheres no setor de TI do país: $24,05 \%$ para $19,83 \%$ entre 2007 e 2017 . Este fato tem despertado a atenção mundial por meio de governos, empresas e instituições de ensino [PISA, 2015], a fim de que estratégias sociais possam intervir a fim de promover a diversidade de gênero na área de TI.

No universo educacional brasileiro observa-se que o número de mulheres matriculadas em cursos de computação, no nível superior, tem sofrido também diminuição [Medeiros et al., 2012]. Dados do Instituto Nacional de Estudos e Pesquisas Educacionais [INEP, 2015] revelam que, desde 1991 no Brasil, as mulheres matriculadas em cursos da área de computação diminuíram 19,37\%, representando então 15,53\% do número de alunos matriculados no curso de Ciência da Computação.

Medeiros (2005), que é um ativista em discussões e pesquisas sobre gênero e computação, afirma que a sociedade brasileira precisa estimular as mulheres a se tornarem agentes ativas em uma sociedade movida pela informação, tecnologia e inovação. Para este autor, alguns pilares precisam ser trabalhados a fim de que mais mulheres possam ser absorvidas pela área de tecnologia como: (i) reconsiderar a estrutura educacional; (ii) desenvolver novos conteúdos, e (iii) considerar as estratégias educacionais que atendam as meninas ainda em sua formação básica na infância. Desses pilares, observam-se pesquisas que já vêm rompendo barreiras quanto à equidade de gênero no Brasil como as desenvolvidas mais recentemente por: [Frigo et al., 2020], [Santana et al., 2020], [Aires et. al., 2018], [Martins et al., 2019].

Dessa maneira, este artigo visa apoiar a introdução de jovens meninas (faixa etária de 11 a 16 anos) na área da computação, através de uma iniciativa que desenvolva o pensamento computacional. O principal objetivo desta iniciativa é apresentar a área da computação, especialmente conceitos da programação de computadores, através de histórias reais ou fictícias. Espera-se discutir, estruturas programáveis nos cenários das histórias trazidas pelas próprias crianças, mantendo o interesse técnico e emocional das meninas em programar as narrativas que chamam a sua atenção.

Esta iniciativa é desenvolvida através de ações do projeto de extensão StoryGirl, conduzido em parceria por duas instituições públicas federais no Rio de Janeiro, atendendo meninas de escolas pública e privada. Nele, é aplicado o Scratch, linguagem de programação em blocos de fácil orientação para meninas na faixa-etária alvo desta iniciativa. Objetivamente, este artigo apresenta os primeiros resultados alcançados em três estudos iniciais da estratégia proposta.

Este artigo é organizado considerando a Seção 2 com a discussão do arcabouço científico nacional sobre o pensamento computacional, destacando o ensino da programação de computadores a partir de uma linguagem de programação em blocos, para crianças e adolescentes. Na seção 3, a construção e estrutura do Projeto StoryGirl são apresentados. Na Seção 4 são tratadas as aplicações e detalhamento sobre o preparo e execução das oficinas do projeto. As discussões sobre os resultados da investigação são relatadas na Seção 5. Na conclusão, são apresentadas as contribuições do estudo e os próximos passos desta iniciativa frente às atividades do projeto de extensão e à pesquisa científica associada. 


\section{Ensino da Programação de Computadores para Crianças e Adolescentes}

Pesquisas recentes discutem as contribuições de iniciativas que fomentem a inserção de mulheres nas áreas que envolvem o STEM [Aono et al., 2017], [Franco et al., 2018], [Santos e Oliveira, 2020]. Muitas delas defendem o design e execução de estratégias que atinjam meninas em sua fase de vida infanto-juvenil. Isso ocorre porque acredita-se que influências sociais e culturais contribuem para o desenvolvimento cognitivo e novos saberes das crianças.

A literatura mostra que a linguagem de programação em blocos tem sido amplamente utilizada com o público infantil com o objetivo de desenvolver $o$ pensamento computacional em crianças [França e Tedesco, 2019]. Aono et al. (2017), Rodriguez et al. (2015), discutem projetos cujo objetivo é o desenvolvimento do pensamento computacional em crianças através da programação de jogos em Scratch. Nessas pesquisas existe uma discussão efetiva sobre a inserção de competências computacionais ainda em nível educacional básico e médio na intenção de diminuir as dificuldades sofridas pelas crianças na área de exatas que é um dos responsáveis pelo abandono de alunos dos cursos de computação na universidade. Esses autores defendem que quanto mais cedo os indivíduos desenvolvem competências lógicas, mais natural se torna o processo de aprendizagem da área de exatas e consequentemente das áreas de tecnologia. Outras pesquisas com foco no pensamento computacional também têm se destacado quanto ao desenvolvimento de competências acadêmicas que não necessariamente estão relacionadas ao desenvolvimento de software e jogos por crianças [Mattos et al., 2017], [Schneider et al. 2019], [Junior et al. 2019].

Há também pesquisas que discutem o desenvolvimento do pensamento computacional no público feminino, que tem sofrido com uma baixa presença na área de computação [Marquiori et al., 2019]. Traçando o mapeamento simplificado de estratégias que conduzem o ensino da programação de computadores em Scratch através de narrativas (uso da técnica Storytelling), pode-se destacar [Farias et al., 2019] e [Gomes et al., 2017]. O diferencial trazido por este artigo está em envolver crianças e adolescentes (entre 11 e 16 anos) na programação de computadores, tendo como estrutura de aprendizado a programação de cenários de histórias de interesse da criança participante. Dessa maneira, a estrutura ensino-aprendizado-prática é individualizada de forma lúdica e divertida. Apesar de cada criança trabalhar em sua história de interesse, o projeto estimula a troca de conhecimento entre as participantes, construindo um ambiente colaborativo e acolhedor.

\section{Desenvolvendo o Pensamento Computacional através de Histórias}

As iniciativas envolvidas no Projeto StoryGirl focam na inserção de meninas entre 1116 anos de escolas públicas e privadas no universo STEM. Para isso, busca-se desenvolver competências lógicas de programação através de narrativas de histórias de interesse desse público. Entende-se que estas ações podem promover o desenvolvimento do raciocínio lógico e pensamento computacional ainda na fase primária de educação das meninas, estimulando este gênero no interesse às áreas exatas. Este projeto visa também contribuir com a transformação cultural das meninas, estimulando o interesse pela área de exatas e fomentando a entrada e a formação de meninas nos cursos de graduação em áreas afins ao projeto. 
O programa articula ações de pesquisa, onde serão estudadas as formas mais indicadas de estruturar e ensinar através de histórias os conteúdos lógicos de programação de forma colaborativa. Através do uso e adaptação de técnicas de visualização existentes, espera-se chegar a soluções com explicações intuitivas e que aumentem a compreensão das meninas sobre as estruturas lógicas contidas em suas histórias. Com estas articulações propostas, visa-se atender o desenvolvimento computacional de meninas através de dinâmicas que as estimulem a automatizar soluções por meio do pensamento algorítmico; identificar, analisar e implementar soluções [Barr et al., 2011].

Durante o desenvolvimento do projeto são conduzidos oficinas e debates sobre o papel das mulheres na ciência. São produzidos meios de disseminação do pensamento lógico, introduzindo soluções programáveis e apresentando técnicas de programação em linguagens de fácil entendimento para crianças como o Scratch. Espera-se trabalhar com histórias que refletem as experiências dessas meninas no âmbito lúdico, social ou escolar. Com base nos cenários programados sobre as experiências pessoais das meninas, espera-se projetar futuramente um ambiente colaborativo de troca de conhecimentos. Resumidamente, o presente projeto é motivado por: (a) Influenciar meninas nas áreas envolvidas no STEM; (b) Habilitar meninas a extraírem conhecimento de histórias através dos cenários programados; (c) Propor iniciativas que envolvam estratégias de ensino-aprendizagem de programação através de cenários em narrativas; (d) Capacitação de alunos da graduação para atuação como tutores das meninas do ensino básico conduzida pela coordenação; (e) Produção de material base de preparação da tutoria e das participantes do projeto, pela coordenação e tutoras respectivamente.

\subsection{Estrutura da Ações do Projeto}

Este projeto é organizado em quatro fases que serão executadas ciclicamente a cada nova turma de crianças atendida (Figura 1). O objetivo é que a cada nova iteração, os artefatos produzidos sejam melhorados e as dinâmicas de condução das oficinas com as crianças sejam aprimoradas.
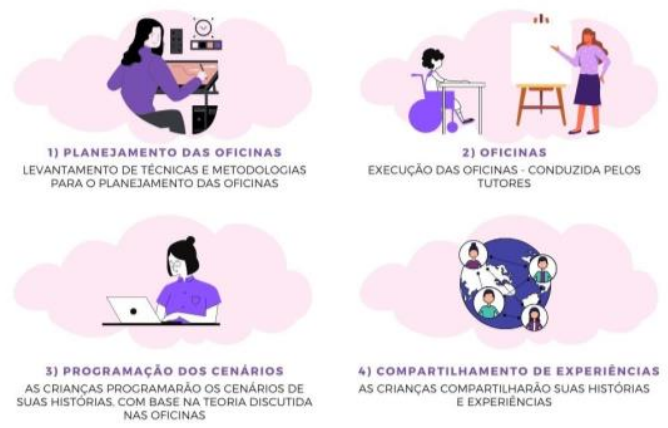

Figura 1. Estrutura do Projeto.

Na Fase I é prevista a execução do planejamento das oficinas e o treinamento dos tutores. Nesta fase, as oficinas são projetadas e preparadas de acordo com o público (número de participantes e perfil das alunas) e os materiais são produzidos. Os tutores do projeto são alunas(os) do curso de graduação na área de computação. Na Fase II é conduzida a oficina, permitindo uma interação direta entre tutores e participantes. Ainda na Fase II, as estruturas básicas de programação são apresentadas através de exemplos 
práticos, troca de ideias e muito acolhimento. A Fase III prevê a prática dos conceitos discutidos na oficina teórica. Nesta fase, os participantes são convidados a praticarem a teoria apresentada na programação dos cenários de suas respectivas histórias. Por fim, a Fase IV fomenta o compartilhamento das experiências técnicas e sociais adquiridas na sessão a partir da troca entre as crianças.

\section{Aplicação das Ações do Projeto}

O projeto começou a ser pensado em 2019, mas ganhou forma em 2020. Como é conhecido, o ano de 2020 foi atípico e mudanças foram necessárias quanto ao desenvolvimento das atividades do projeto. Neste ano, foram concentradas as atividades de produção do material da oficina e preparação dos tutores. Ainda em 2020 aconteceu um teste piloto com cinco crianças isoladamente para testar o material produzido. Já em 2021 o projeto foi conduzido em uma primeira turma oficial (com alunas de escolas privadas), não associada diretamente a uma escola e, posteriormente, executada com uma turma de uma escola pública. É importante destacar que em função da pandemia, todas as atividades que aconteceram a partir de 2020 até o momento foram no ambiente online.

\subsection{Estudo Piloto}

A primeira iniciativa para a avaliação da estrutura do projeto e material produzido para as oficinas aconteceu em 2020. A partir dela, foi investigada a viabilidade para explorar na teoria e prática os conceitos das estruturas básicas da lógica de programação. Neste estudo, 6 crianças foram convidadas a relatarem suas histórias reais e três delas foram programadas em Scratch. As histórias, neste estudo piloto, foram organizadas pelas tutoras deste projeto a fim de investigar as potencialidades das histórias reais em explorar recursos básicos da programação em blocos. As histórias programadas exploraram conceitos envolvidos com a programação básica de computadores, como: orientação dimensional, proporção da imagem, tempo de exposição da cena e diálogo associado. Esta primeira avaliação deixou clara a possibilidade de explorar as estruturas simples de programação em histórias.

É importante destacar que esta primeira iniciativa de investigação da viabilidade do projeto ocorreu considerando as histórias fornecidas por crianças na faixa etária esperada no projeto. Vale ressaltar, que as crianças não programaram os seus respectivos cenários na prática. Elas apenas os viram prontos. Os resultados desta avaliação mostram como promissoras o uso de narrativas para explorar as estruturas básicas de orientação e proporção lógicas com base nas percepções das tutoras e coordenadoras do projeto. A fase seguinte do projeto buscou investigar se as crianças e adolescentes, participantes da oficina de programação, são capazes de programar suas próprias histórias, tendo como suporte em seu processo de aprendizagem os demais participantes da oficina e os tutores envolvidos no projeto.

\subsection{Planejamento e Execução das Oficinas do Projeto}

Nesta fase do estudo, foram aplicadas duas oficinas, com durações, conteúdos e públicos diferentes. Na primeira oficina, houve a participação de 8 meninas, entre $11 \mathrm{e}$ 15 anos, alunas do $6^{\circ}$ ao $1^{\circ}$ ano do Ensino Médio de diferentes escolas do setor público e privado do Rio de Janeiro. Esta oficina ocorreu em formato online, dividida em dois encontros com duas horas de duração em março de 2021. Já na segunda oficina, houve a 
participação de 10 meninas entre 14 e 16 anos, alunas do $9^{\circ}$ ano do ensino fundamental de uma escola pública do Rio de Janeiro. Essa oficina também ocorreu em formato online, em um encontro de 2 horas.

\section{Produção do material para as oficinas:}

O material de apoio para as oficinas foi produzido na fase de preparação do projeto e com o foco nas meninas. A cada nova oficina o material é atualizado com base no tempo disponível e no perfil das participantes.

O material usado na oficina foi baseado em slides, com conteúdo produzido em linguagem simples e objetiva. Os conteudistas das oficinas optaram por uma breve introdução e contextualização dos conceitos a serem abordados, apoiados em figuras lúdicas para estimular e manter a atenção das meninas participantes. Foram apresentados o histórico feminino frente à área da computação, e também os conceitos principais da computação e sua relevância no universo de TI com uso do Scratch. Após a contextualização, foram apresentados os conceitos de algoritmo em um exemplo básico e com alto nível de abstração.

\section{Oficina 1:}

As meninas participantes foram convidadas individualmente. A escolha deste grupo de participantes foi baseada nos seguintes critérios: (i) meninas com conhecimento prévio na linguagem de programação em blocos Scratch; (ii) meninas estudantes no ensino privado no Rio de Janeiro; (iii) meninas com disponibilidade para participar da oficina em dois encontros de duas horas.

No primeiro dia da oficina foram apresentados conceitos básicos da programação de computadores como: (i) repetição e condição, (ii) sequência lógica de ação, (iii) cenários, (iv) variáveis, (v) interação entre objetos, (vi) eventos e (vii) atores. Além disso, foram conduzidas discussões sobre a inserção de mulheres na área da computação, e onde a programação de computadores é observada no dia a dia de todo indivíduo. No segundo encontro, as participantes apresentaram seus projetos e discutiram as facilidades e dificuldades no processo da programação em blocos. Fez parte do conteúdo trabalhado na oficina apresentar a linguagem Scratch e suas potencialidades, destacando seus recursos básicos como personagens e blocos estruturais. No primeiro encontro com as meninas foram apresentados 3 exemplos práticos, e através deles foram trabalhados os conceitos básicos da programação já citados.

A Figura 2 apresenta um dos exemplos trabalhados na oficina. Seu objetivo é difundir o conceito de atores, cenários, eventos e repetições através das falas do gatinho "Olá" e "Tudo bem?". Entre as falas existia também um espaço temporal de 2 segundos, assim que a bandeira fosse clicada, ou seja, a partir do início de um novo evento. Nesta fase da oficina, foram trabalhados também a caracterização dos atores e os recursos do Scratch para esta finalidade. A Figura 3 mostra um diálogo de ímpar e par, onde foram trabalhadas estruturas de sequência lógica, eventos e condicionais. 


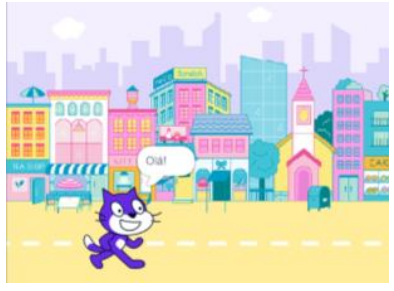

Figura 2. Conceitos de Atores.

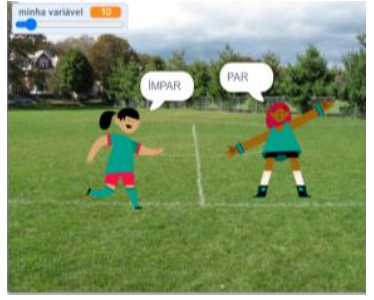

Figura 3. Uso dos conceitos de Variável.

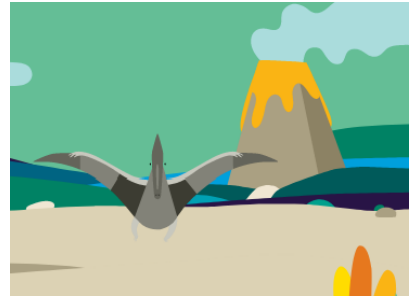

Figura 4. Uso do conceito de repetição.

No segundo encontro da primeira oficina, as meninas iniciaram a sua participação informando a temática de suas histórias. Em seguida, selecionaram um ou mais cenários da história a serem programados e desenvolveram a programação em Scratch. A oficina conduzida proporcionou um ambiente de produção coletiva, onde as meninas e tutoras acompanharam o ciclo de aprendizagem desde a mediação, apoio em dúvidas de forma individual ou coletiva, e desenvolvimento de novas capacitações nas meninas participantes. Para fortalecer o conhecimento adquirido pela oficina, foram realizadas apresentações das histórias programadas pelas próprias meninas, destacando as estruturas lógicas exploradas.

\section{Oficina 2:}

A segunda oficina foi conduzida em uma escola do ensino público do estado do Rio de Janeiro. As meninas participantes foram selecionadas pela diretora da escola considerando: (i) alunas que tinham disponibilidade para participar da oficina em um encontro de duas horas; (ii) alunas com infraestrutura mínima (computador, tablet ou celular com acesso à internet) em casa para participar da oficina; (iii) alunas sem conhecimento prévio em Scratch.

A oficina foi organizada em duas etapas, onde cada uma teve duração de 1 hora. $\mathrm{Na}$ primeira hora foi considerada a ambientação das participantes na temática da oficina, e foram apresentados conceitos básicos da programação de computadores: (i) repetição e condição, (ii) sequência lógica de ação, (iii) cenários, (iv) eventos e (v) atores. Diferente da oficina 1, a oficina 2 trabalhou conceitos ainda mais introdutórios, em razão do tempo menor de disponibilidade da escola para esta ação. A segunda hora foi reservada para a prática dos conceitos e uma tarefa envolvendo as histórias de cada participante. Fez parte do conteúdo trabalhado na oficina apresentar a linguagem Scratch e suas potencialidades, destacando em dois exemplos, seus recursos básicos como personagens e blocos estruturais. O exemplo escolhido traduziu o conceito de repetição na movimentação das asas de um pássaro (Figura 4).

Ao final da oficina 2, foram conduzidas discussões sobre a carreira na área de tecnologia e possíveis caminhos a serem seguidos para alcançar uma formação. A oficina instigou a curiosidade das participantes, mesmo naquelas que não conseguiram entregar a tarefa, e gerou perguntas relevantes a respeito dos caminhos possíveis em áreas que envolvam a computação.

\section{Resultados Alcançados e Discussões}

Os primeiros resultados alcançados pelo projeto mostram ser possível introduzir conceitos lógicos de programação de computadores através de histórias de forma leve, 
lúdica e divertida. Esta afirmativa está baseada no feedback das participantes e também na análise das produções desenvolvidas na oficina.

A fim de consolidar a premissa que defende a necessidade por instruções bem definidas para o processamento de uma atividade por uma máquina, foi proposto em ambas as oficinas que as meninas orientassem verbalmente o tutor na construção de um coração, considerando os quatro quadrantes formados por duas retas. Esta atividade permitiu a interação direta entre as meninas e a tutora, atuando como uma atividade "quebra-gelo" entre elas. Nela, o papel da tutora foi de atuar como interface entre as meninas com o computador, que apenas seguia instruções, e as meninas exerciam o papel de programadoras, instruindo o que deveria ser feito. $\mathrm{O}$ resultado é apresentado nas Figuras 5 e 6.

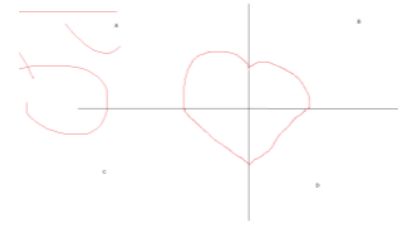

Figura 5. Resultado da atividade na primeira oficina.

\section{Figura 6. Resultado da atividade na segunda oficina.}

Era, portanto, objetivo desta atividade simular a produção de instruções claras e precisas pelas participantes, preparando-as para a atividade seguinte da oficina. A atividade sobre instruções foi realizada com sucesso. Após alguns fracassos, o coração foi formado pela turma de meninas participantes. Em ambas as oficinas, as meninas concluíram que precisavam ser específicas em suas instruções, do contrário as instruções não seriam reconhecidas pelo computador.

As histórias programadas em Scratch nas oficinas mostraram que as meninas foram capazes de pôr em prática alguns dos conceitos envolvidos nas discussões do pensamento computacional. A Figura 7 apresenta um frame da narrativa programada por uma das meninas participantes da primeira oficina. Em sua história, cujo título é "Bichos verdes", é narrada a trajetória do Pequeno Goblin, um personagem que queria ir ao Canyon mas o Centauro não deixava porque ele não tinha o tamanho ideal. Com isso, ele começava a sua pequena jornada para conseguir realizar sua vontade de visitar aquele lugar tão desejado e acaba visitando um mago que, através de um feitiço, transforma o personagem principal em outra pessoa, uma pessoa maior. No final do conto, o pequeno Goblin retorna ao centauro e pede passagem, dessa vez, obtendo resposta positiva e realiza seu desejo.

Para a programação dessa história, a menina utilizou 5 cenários e 4 personagens, com um nível de complexidade relevante. Em seus códigos, ela conseguiu estruturar sua história de maneira lógica, com conceitos de espera (que envolvem uma certa dificuldade no cálculo de tempo, para manter a sincronia dos personagens), também utilizou os comandos de esconder e mostrar, comandos de coordenadas cartesianas para as posições dos personagens. Um exemplo da programação usada pela menina pode ser acompanhado na Figura 8. A participante também explorou a caracterização dos personagens (atores) e usou uma estrutura de repetição. As demais participantes da oficina 1, apesar de não utilizarem conceitos avançados da computação, conseguiram estruturar suas histórias em sequências lógicas e programar no Scratch. A maioria delas 
decidiu trabalhar com mais de 1 personagem, usaram os conceitos de espera e coordenadas cartesianas.

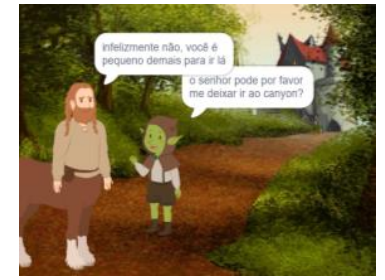

Figura 7. História chamada de "Bichos Verdes".

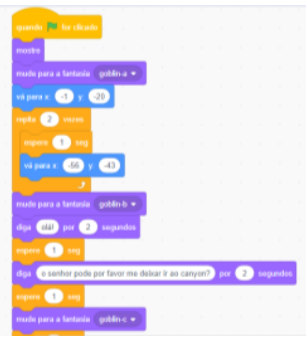

Figura 8. Programação do personagem Goblin.

Na oficina 1 não houve problemas graves de infraestrutura durante o curso, as participantes mantiveram contato com as tutoras entre o primeiro e segundo encontro tirando dúvidas e melhorando suas histórias programadas. Todas as participantes apresentaram sua respectiva tarefa e se mostraram motivadas para continuar em novas iniciativas que evoluam seus conhecimentos na programação.

Já na oficina 2, das 10 participantes, apenas 3 conseguiram produzir e enviar sua tarefa finalizada em formato de vídeo. A principal razão foi a dificuldade de infraestrutura pessoal de cada participante, uma vez que estavam em suas casas. Em geral, a internet ou os dispositivos eletrônicos das participantes não suportaram as interações em vídeo chamada pelo Google Meeting (plataforma usada na condução das oficinas) e a execução simultânea das atividades no Scratch.

A Figura 9 representa um frame da história programada por uma das participantes da segunda oficina. Em sua história, é narrado um diálogo do personagem Giga, que reclama sobre a falta de atividades para fazer e que, para se distrair, propõe uma amizade com o usuário. Para a programação dessa história, a participante usou 1 cenário e 1 ator, juntamente com elementos de som, movimento, controle e aparência do Scratch. Além disso, ela utilizou o conceito de sequência lógica, sabendo controlar o fluxo da história (Figura 10).

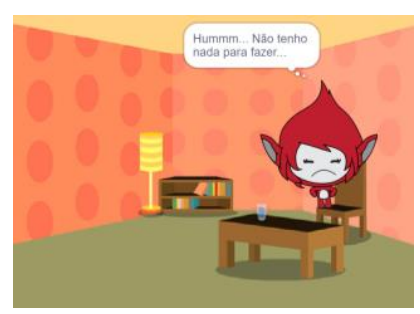

Figura 9. História "Amigos".

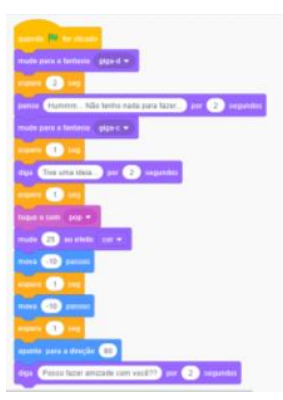

Figura 10. Programação das ações e eventos da história "Amigos".

$\mathrm{Na}$ condução de ambas as oficinas, ficou claro que o conhecimento e experiência anteriores das participantes impactou nos resultados. Ficou claro também que a infraestrutura é necessidade básica para esta oficina, seja para o desenvolvimento das 
tarefas práticas, seja para a interação entre participantes e tutoras. No entanto, o interesse mostrado pelas participantes, antes, ao longo das oficinas, e depois da sua execução foi o mesmo em ambos os perfis trabalhados. Ficou claro que as meninas se envolveram ativamente em suas histórias e que até mesmo aquelas que não conseguiram desenvolver as suas por razões técnicas, comentaram e indicaram melhorias nas histórias programadas de seus pares.

\section{Conclusão}

Este artigo apresentou os resultados obtidos na aplicação das duas oficinas do projeto que visa estimular o pensamento computacional em meninas na faixa-etária de 11 a 16 anos. Ele tem por objetivo apresentar a programação de computadores através da linguagem em blocos Scratch, incentivando a inclusão do gênero feminino em áreas envolvidas no STEM. O diferencial desta ação é a introdução de conceitos básicos da programação de uma maneira simplificada, através de histórias que envolvam as participantes. O resultado atingido com as oficinas mostrou interesse das meninas na área da computação, despertando nas participantes: (i) o sentimento de investigação a respeito da área e suas potencialidades; (ii) o desejo de continuar a participar de novas oficinas sobre computação; (iii) a indicação da oficina para outras meninas interessadas.

Como ponto de atenção sobre os resultados observados, destacam-se a desigualdade de conhecimento das áreas de exatas entre as participantes do segmento público e privado. Destaca-se a interferência da infraestrutura tecnológica de cada menina em sua participação ativa na oficina.

Como próximos passos desta iniciativa, espera-se conduzir a oficina em mais turmas de escolas do setor público e privado, no formato online ou presencial, a fim de atingir mais meninas e desenvolver nelas o pensamento computacional. Esta iniciativa pode ser conduzida e ampliada a partir de uma parceria com professores de matemática, história etc. Ainda como desdobramento dessa iniciativa, espera-se criar uma rede colaborativa de apoio técnico e social com base nas histórias programadas.

\section{Agradecimentos}

Esta pesquisa recebeu apoio da FAPERJ - E-26/211.367/2019 (248406). Os agradecimentos também são estendidos para os participantes desse estudo.

\section{Referências}

Aires, J., Mattos, G., Oliveira, C., Brito, A., Aragão, A. F., Alves, S., ... \& Moreira, G. (2018, July). Barreiras que impedem a opção das meninas pelas ciências exatas e computaçao: Percepçao de alunas do ensino médio. In Anais do XII Women in Information Technology. SBC.

Aono, A., Rody, H., Musa, D., Pereira, V., \& Almeida, J. (2017). A Utilização do Scratch como Ferramenta no Ensino de Pensamento Computacional para Crianças. In Anais do XXV Workshop sobre Educação em Computação. Porto Alegre: SBC. doi:10.5753/wei.2017.3556

Barr, D., Harrison, J., Conery, L. (2011). Computational thinking: A digital age skill for everyone. Learning \& Leading with Technology 38, 6 (2011), 20-23 
Farias, C., da Cruz, V. G., Farias, J. S., Braz, D. C., Brito, B. M., \& de Souza Carvalho, A. (2019, November). Estimulando o Pensamento Computacional: uma experiência com Scratch. Jr. In Anais do Workshop de Informática na Escola (Vol. 25, No. 1, p. 197).

França, R., \& Tedesco, P. (2019, November). Pensamento Computacional: Panorama dos Grupos de Pesquisa no Brasil. In Brazilian Symposium on Computers in Education (Simpósio Brasileiro de Informática na Educação-SBIE) (Vol. 30, No. 1, p. 409).

Franco, A. A., da Costa, A. C. M., Graça, R. J. D. S., do Nascimento, T. C., Rosa, T. S., \& Muniz, V. H. J. (2018). Lugar de Aluna de Ensino Médio é na Programação. Por que não?.

Frigo, L. B., Moro, F. F. ,Padilha, R. O.,Pozzebon , E.. Meninas em Ação Atividades Inspiradoras para Projetos Parceiros do Programa Meninas Digitais. In: Anais do XI Women In Information Technology (WIT 2020), Congresso da Sociedade Brasileira de Computação (CSBC 2020). Disponível em: https://sol.sbc.org.br/index.php/wit/article/view/11276. Acessado em: 15 de Abril de 2021.

Gomes, T., Castro, F., \& Tedesco, P. C. A. R. (2017). Desenvolvendo o Pensamento Computacional na Educação Infantil: Um toolkit educacional sobre conceitos de programação baseado em storytelling transmedia. Sánchez, J, 31-40.

INEP. 2015. Resumo Técnico da Educação Superior 2013. Diretoria de Estatísticas Educacionais DEED, Instituto Nacional de Estudos e Pesquisas Educacionais Anísio Teixeira, Brasília-DF, 82

Jornal da USP. (2018). Por que as mulheres "desapareceram" dos cursos de computação?. Disponível em: http://jornal.usp.br/universidade/por-que-as-mulheres-desapareceram-doscursos-de-computacao/ .Acessado em: 05/06/2021.

Junior, B., Cavalheiro, S., \& Foss, L. (2019, November). Revisitando um Jogo Educacional para desenvolver o Pensamento Computacional com Gramática de Grafos. In Brazilian Symposium on Computers in Education (Simpósio Brasileiro de Informática na EducaçãoSBIE) (Vol. 30, No. 1, p. 863).

Marquiori, V., Oliveira, M., \& Nascimento, G. (2019, July). Letramento de Meninas em Programação através do Pensamento Computacional para Compreensão de Problemas. In Anais do XIII Women in Information Technology (pp. 109-113). SBC.

Mattos, M., de Araújo, L., Zucco, F., Hein, N., da Cunha, K., de Oliveira, G., ... \& Bursoni, P. (2017, July). Aplicação da prática colaborativa no desenvolvimento de um jogo para o ensino da programação. In Anais do XIV Simpósio Brasileiro de Sistemas Colaborativos (pp. 21-30). SBC.

Martins, A., Silva, J., Santos, J., \& Rebouças, A. (2019, July). Fatores que Atraem e Afastam as Meninas de cursos da Área de TI. In Anais do XIII Women in Information Technology (pp. 114-118). SBC.

Medeiros, C. B. (2005). From subject of change to agent of change: women and IT in Brazil. In Proc. of the International Symposium on Women and ICT: creating global transformation (CWIT '05), C. Morrell and J. Sanders (Eds.). ACM, New York, NY, USA, Article 15.

Medeiros, C., Coelho, R., e Maciel, C. (2012). Participação Feminina na Computação. Computação Brasil. 19, 28-29.

PISA. 2015. O que está por trás da desigualdade de gênero na educação? OCDE. Acessado em $15 / 04 / 2021$.

Rodriguez, C., Zem-Lopes, A. M., Marques, L., \& Isotani, S. (2015, October). Pensamento Computacional: transformando ideias em jogos digitais usando o Scratch. In Anais do Workshop de Informática na Escola (Vol. 21, No. 1, p. 62). 
Santana, T. S.; Braga, A. H. Uma Análise Cienciométrica das Publicações do Congresso da Sociedade Brasileira de Computação na Perspectiva das Mulheres na Computação. In: Anais do XIV Women In Information Technology (WIT 2020), Congresso da Sociedade Brasileira de Computação (CSBC 2020). Disponível em: https://sol.sbc.org.br/index.php/wit/article/view/11310. Acessado em: 15 de Abril de 2021.

Santos, C., \& Oliveira Filho, A. (2020). Robótica e Interdisciplinaridade: Aprendizagem Criativa Atraindo Meninas para a Tecnologia. In Anais do XIV Women in Information Technology, (pp. 120-128). Porto Alegre: SBC. doi:10.5753/wit.2020.11282

Schneider, G., Bernardini, F., \& Boscarioli, C. (2019, November). Ensino do Pensamento Computacional por meio de Internet das Coisas: Possibilidades e Desafios. In Brazilian Symposium on Computers in Education (Simpósio Brasileiro de Informática na EducaçãoSBIE) (Vol. 30, No. 1, p. 169).

Silva, Elizandra Silveira. Mulheres na informática. Anais do Congresso Nacional Universidade, EAD e Software Livre, v. 2, n. 8, 2017. Disponível em: http://www.periodicos.letras.ufmg.br/index.php/ueadsl/article/view/12786. Acesso em: 2 jun. 2021.

Softex. (2019) Atuação da Mulher no Mercado de Trabalho Formal Brasileiro em Tecnologia da Informação. Arquivo eletrônico disponível em: https://www.ftp.softex.br/Inteligencia/mulheres_na_ti/mulheres_na_ti.pdf. Acessado em: Março 2021. 